\title{
An Asymptotic Expansion for the Recharge-Discharge Model of ENSO
}

\author{
Sulian Thual, Boris DewitTe, And Nadia Ayoub \\ Laboratoire d'Etudes en Géophysique et Océanographie Spatiales, Toulouse, France \\ OLIVIER THUAL \\ Université de Toulouse, INPT, CNRS, IMFT, Toulouse, France
}

(Manuscript received 22 August 2012, in final form 6 March 2013)

\begin{abstract}
The dynamics of El Niño-Southern Oscillation (ENSO) in the equatorial Pacific Ocean are largely associated with the slow thermocline adjustment at interannual and basin scales. This adjustment involves, among other things, the fast propagation and reflection of equatorial waves by wind stress forcing. A simple and straightforward asymptotic expansion of the long-wave equations is proposed using the low-frequency approximation. The asymptotic expansion is performed in Fourier space, retaining only the gravest equatorial long waves and baroclinic modes with the largest scale, and considering small dissipation by friction and boundary reflections. This leads to an asymptotic model for the thermocline response to wind stress forcing, which is in essence the ocean component of the recharge-discharge model of ENSO. The asymptotic model is nonheuristic and in broad agreement with some essential results scattered in previous studies. Thermocline variability is divided into a sloping "Tilt mode" that adjusts instantly to wind stress forcing and a zonal-mean "Warm Water Volume mode" that adjusts as a time integrator to wind stress curl. The model has a plausible energy budget and its solutions are in good agreement with observations. Results suggest that the net adjustment rather than the explicit delays of equatorial waves is essential for the slow thermocline adjustment, and this is best described by the recharge-discharge model.
\end{abstract}

\section{Introduction}

The El Niño-Southern Oscillation (ENSO) is, after the seasonal cycle, the most energetic climate fluctuation known on Earth in today's climate, with dramatic ecological and social impacts. Its dynamics in the equatorial Pacific Ocean are largely associated with the slow ocean adjustment, at interannual and basin scale (Neelin et al. 1998; Kessler 2002). In ENSO theory, this ocean adjustment may drive a phase reversal from El Niño to La Niña and inversely, through the propagation and reflection of equatorial waves (Suarez and Schopf 1988) or the recharge-discharge of the equatorial heat content (Jin 1997a,b). This ocean adjustment involves considerable energy exchanges through variations of the equatorial thermocline depth (Goddard and Philander 2000; Brown and Fedorov 2010b), and

Corresponding author address: Sulian Thual, Courant Institute of Mathematical Sciences, New York University, 251 Mercer Street, New York, NY 10012.

E-mail: sulian.thual@gmail.com explains the relatively high predictability of ENSO (Cane and Zebiak 1985; Meinen and McPhaden 2000; McPhaden 2012).

Various studies describe analytically this slow ocean adjustment, with various degrees of complexity. Some of those studies consider in particular a low-frequency approximation to expand the long-wave equations (Jin 1997a,b; Fedorov 2010; Clarke 2010). This permits integration of, on an interannual time scale, fast processes of the equatorial Pacific Ocean. Jin (1997a) has illustrated the major recharge-discharge paradigm of ENSO in a conceptual model, with the long-wave equations averaged into a western and eastern box of the equatorial Pacific, and where, in the low-frequency approximation, the propagation time of the equatorial Kelvin wave is omitted. Jin (1997b) has further derived a stripped-down coupled model with a collective representation of equatorial waves, exhibiting in the low-frequency approximation an ENSO regime with essential physics from Jin (1997a). Fedorov (2010) has considered the lowfrequency approximation to expand the long-wave equations into power series. This allows describing 


\section{TILT}
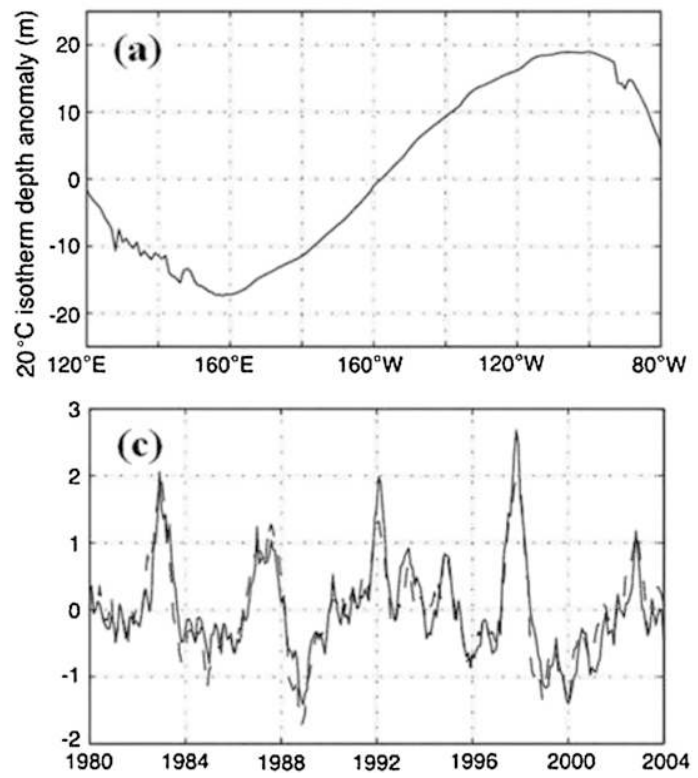

WWV
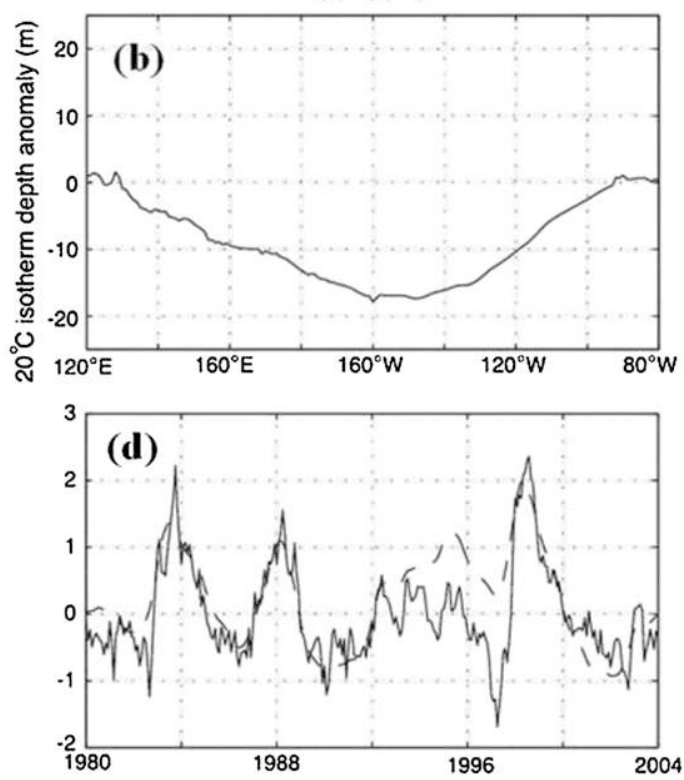

FIG. 1. (a) First EOF (51\% of variance) of the $20^{\circ} \mathrm{C}$ isotherm depth anomaly $(\mathrm{m})$ in the equatorial Pacific Ocean $\left(5^{\circ} \mathrm{N}-5^{\circ} \mathrm{S}\right)$ and $(\mathrm{c})$ corresponding first principal component (solid line). The dashed curve in (c) is the Niño-3.4 SSTA time series (correlation $r$ is 0.93 ). (b) Second EOF (35\% of variance) and (d) the corresponding second principal component (solid line). The dashed curve in (d) is the time-integrated Niño-3.4 SSTA time series. Time series are normalized at a variance of 0.5 [from Clarke et al. (2007)].

ENSO with a rigorous and rich integro-differential equation, which encompasses paradigms from Suarez and Schopf (1988) and Jin (1997a,b). Clarke (2010) has shown that, in the low-frequency approximation, the Fourier solutions to an interannual wind stress forcing decompose into a tilting and shallowing/deepening component of thermocline anomalies.

At interannual time scales, thermocline variability can be described by the evolution of two zonal modes of basin scale, the so-called "Tilt" and "Warm Water Volume" (WWV) modes (Meinen and McPhaden 2000). The Tilt and WWV modes can be statistically inferred from the first and second EOF modes of observed thermocline depth anomalies, as shown in Fig. 1 [from Clarke et al. (2007)]. They consist of a zonal tilting and a homogeneous shallowing/deepening of the equatorial thermocline, respectively. The associated time series show that the Tilt and WWV modes are in phase with sea surface temperatures (SST) and time-integrated SST anomalies (SSTA), respectively. Because at interannual time scales SST anomalies are almost in phase with wind stress anomalies, the Tilt mode adjusts instantly to the wind stress forcing, while the WWV mode adjusts as a time integrator. The WWV mode has been shown to adjust more particularly to wind stress curl (Clarke et al.2007). The evolution of those modes is well described by the long-wave equations (e.g., Bosc and
Delcroix 2008), and more particularly by the rechargedischarge model (Jin 1997a,b). The evolution of the WWV mode is, however, also known to be controlled in part by diathermal transport (Clarke et al. 2007; Brown and Fedorov 2010a; Lengaigne et al. 2011).

In this article, we propose a novel asymptotic expansion of the long-wave equations in the low-frequency approximation, similar to the one of Fedorov (2010). The asymptotic model is, in essence, the ocean component of the recharge-discharge model (Jin 1997a,b), though with some notable differences, and is in broad agreement with some essential results of other studies (e.g., Clarke et al. 2007; Fedorov 2010; Clarke 2010). While some of those studies are intended to describe the full ENSO cycle, our focus here is on their treatment of the slow thermocline adjustment to wind stress forcing. For clarity, the asymptotic model will be denoted hereafter as TW model (for Tilt and WWV model).

The article is organized as follows. Section 2 presents the initial long-wave equations. Section 3 presents solutions without wind stress forcing and without the low-frequency approximation. Section 4 presents the low-frequency approximation and the asymptotic expansion. Section 5 presents the TW model and its solutions. Section 6 compares the TW model solutions with observations. Section 7 is a discussion with concluding remarks. 


\section{Long-wave equations}

We consider linearized shallow-water dynamics in the equatorial Pacific Ocean with the long-wave approximation: the long-wave equations [see Clarke (2008), chapter 3]. The system is truncated at the three gravest baroclinic modes $(n=1,2,3)$ and at the equatorial Kelvin and first-order Rossby wave (e.g., An and Kang 2000; Dewitte 2000; Thual et al. 2011). For each baroclinic mode $n$, the system evolution reads as follows:

$$
\begin{gathered}
\left(\partial_{t}+\varepsilon_{n}\right) K_{n}+c_{n} \partial_{x} K_{n}=c_{n} F_{K-n}, \\
\left(\partial_{t}+\varepsilon_{n}\right) R_{n}-c_{n} \partial_{x} R_{n} / 3=c_{n} F_{R-n}, \\
F_{K-n}=P_{n} \frac{\int_{-\infty}^{+\infty} \tau \varphi_{0} d y}{2 \rho l_{n} c_{n}^{2}}, \text { and } \\
F_{R-n}=P_{n} \frac{\int_{-\infty}^{+\infty}\left(\tau \varphi_{2} / \sqrt{2}\right) d y-\int_{-\infty}^{+\infty} \tau \varphi_{0} d y}{3 \rho l_{n} c_{n}^{2}},
\end{gathered}
$$

where, for each baroclinic mode $n, K_{n}$ is Kelvin wave amplitude, $R_{n}$ is Rossby- 1 wave amplitude, and $F_{K-n}$ and $F_{R-n}$ are projections of zonal wind stress anomalies $\tau$. A friction coefficient is $\varepsilon_{n}, c_{n}$ is the phase speed of Kelvin waves, and $P_{n}$ is a projection coefficient. The $\varphi_{0}=\psi_{0}\left(y / l_{n}\right)$ and $\varphi_{2}=\psi_{2}\left(y / l_{n}\right)$ are the zero- and secondorder Hermite functions, $\psi_{0}$ and $\psi_{2}$, scaled by the oceanic Rossby radius $l_{n}=\sqrt{c_{n} / \beta}$ (see Clarke 2008). The betaplane parameter is $\beta$ and the ocean density is $\rho$. Parameters of the ocean baroclinic structure are taken from Thual et al. (2011, their Table 1). Equatorial waves reflect at the western boundary $(x=0)$ and eastern boundary $(x$ $=L)$ of the equatorial Pacific. This reads, for each baroclinic mode $n$ :

$$
\begin{aligned}
& K_{n}(0, t)=\gamma R_{n}(0, t) \text { and } \\
& R_{n}(L, t)=\eta K_{n}(L, t),
\end{aligned}
$$

where $\gamma$ and $\eta$ are reflection coefficients, and $L$ is the basin length $(18000 \mathrm{~km})$ that extends from $120^{\circ} \mathrm{E}$ to $80^{\circ} \mathrm{W}$. For boundary conditions of no flow at the eastern boundary and of no net flow at the western boundary, $\eta=1$ and $\gamma=0.5$. Thermocline depth anomalies $H$ are recovered by summing up contributions from all baroclinic modes and equatorial waves:

$H=\sum_{n=1}^{3} h_{n}, \quad$ with $\quad \frac{h_{n}}{s_{n} c_{n}^{2}}=\left(K_{n}+R_{n}\right) \varphi_{0}+R_{n} \frac{\varphi_{2}}{\sqrt{2}}$,

where $s_{n}$ is a scaling factor estimated at the mean thermocline depth (see Dewitte 2000; Thual et al. 2011). (a) $p=0$

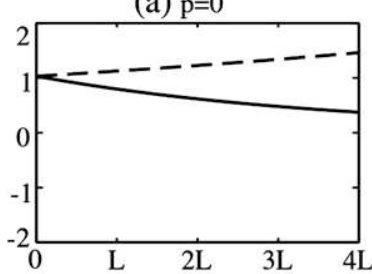

(c) $\mathrm{p}=2$
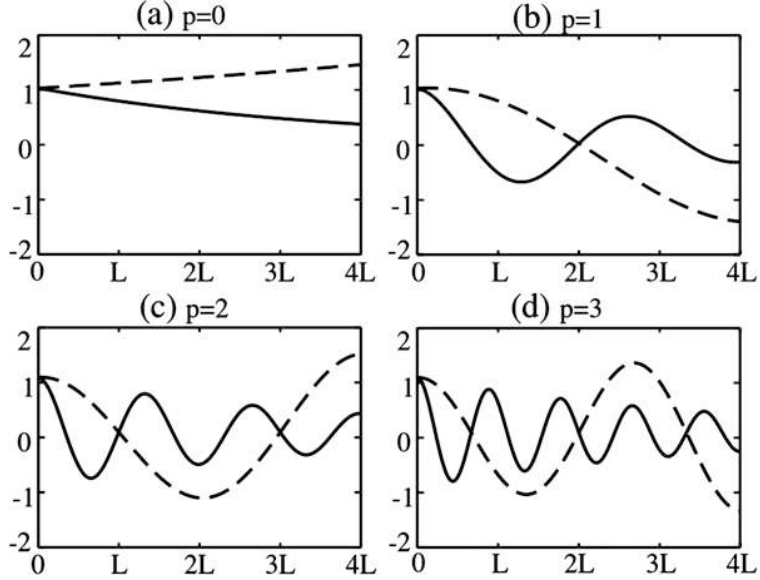

(d) $\mathrm{p}=3$

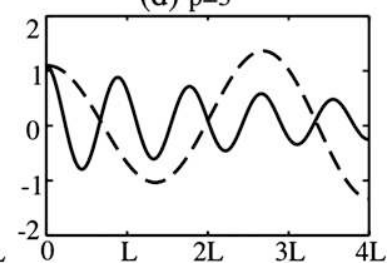

FIG. 2. Solutions of long-wave equations without wind stress forcing at $t=0$ and as a function of longitude $x$. Kelvin (dashed line) and Rossby (thick line) wave, for (a) $p=0$, (b) $p=1$, (c) $p=2$, and (d) $p=3$. For visualization, $x$ extends to four times the basin length $L$. We choose $A_{p}=1, B_{p}=1, \gamma=0.7$, and $\eta=1$.

Hereafter, we will solve the system of Eqs. (1)-(6) independently for each baroclinic mode and omit the subscript $n$ for simplicity.

\section{Solutions without wind stress forcing}

At first, we solve the long-wave Eqs. (1)-(6) without wind stress forcing and without low-frequency approximation. Those solutions are shown for comparison with the asymptotic solutions of Eqs. (1)-(6) that will be presented in the following sections. The elementary solutions read as follows:

$$
\begin{aligned}
K & =\sum_{p} A_{p} \exp \left[-\sigma t-(\varepsilon-\sigma) x / c+i k_{p}(x-c t)\right], \\
R & =\sum_{p} B_{p} \exp \left[-\sigma t+3(\varepsilon-\sigma) x / c-i k_{p}(3 x+c t)\right], \\
\sigma & =\varepsilon-\ln (\gamma \eta) c / 4 L, \quad \text { and } \\
k_{p} & =-p \pi / 4 L,
\end{aligned}
$$

where $p$ is a positive integer, and $A_{p}$ and $B_{p}$ are arbitrary constants satisfying boundary conditions. Figure 2 shows the spatial structure of the four first solutions $(p=0,1$, 2, 3). Similar modes have been described by Jin [1997b, its Eq. (2.7)] and Jin (2001) and designated as free ocean adjustment modes. They can be illustrated by the standing oscillations of an elastic beam with two free boundary conditions. Those solutions are however not valid for high values of $p$, for which the small zonal scales are in contradiction with the long-wave approximation. 
The dissipation rate $\sigma$ in Eq. (10) is identical for all solutions that are indexed by $p$. It accounts for losses by friction $\varepsilon$ and boundary reflections, where over each round-trip propagation across the basin lasting $4 L / c$ the signal is multiplied by $\gamma \eta \leq 1$. The dissipation rate $\sigma$ is of particular interest because it will be encountered in the asymptotic solutions of Eqs. (1)-(6) presented in the following sections.

\section{Low-frequency approximation}

We consider a low-frequency approximation (Jin 1997b; Fedorov 2010; Clarke 2010) to derive asymptotic solutions of Eqs. (1)-(6) in the case of nonzero wind stress forcing. The low-frequency approximation here is an approximation of large spatial scales, of large temporal scales, and of small dissipation. We omit the zonal peculiarities of wind stress forcing and rather consider its basinwide average. Projections of wind stress forcing read $f_{K} / L$ and $f_{R} / L$, where $f_{K}$ and $f_{R}$ are zonally integrated projections:

$$
f_{K}=\int_{0}^{L} F_{K} d x \text { and } f_{R}=\int_{0}^{L} F_{R} d x .
$$

Approximations of large spatial scales have already been made in the initial long-wave Eqs. (1)-(6). The contribution from higher-order Rossby waves is omitted. Those describe smaller meridional scales, as well as smaller zonal scales because of their slow propagations: at first order, we suppose that they are interfering or dissipating with no upscale contribution to the large scale flow. A similar argument is considered to omit the contribution from higher-order baroclinic modes. We nevertheless retain only the three gravest baroclinic modes that we suppose are relevant to the large-scale flow (Dewitte 2000; Thual et al. 2011). The system oscillation frequencies $\omega$ are supposed to be small relative to the frequency $c / 4 L$ of a round-trip propagation across the basin: $\omega \ll c / 4 L$. Finally, the temporal dissipation rate of solutions from previous section [cf. Eq. (10)] is supposed to be small: $\sigma \ll c / 4 L$. This implies both small losses by friction: $\varepsilon \ll c / 4 L$, as well as small losses at boundaries: $-\ln (\gamma \eta) \ll 1$. To achieve this last condition, we consider near-perfect reflections (i.e., near no-flow conditions) at both the western and eastern boundaries: $\gamma \approx 1$ and $\eta \approx 1$. The row of approximations retained here gives us an asymptotic path in parameter space, which we will follow to choose an asymptotic expansion of the system from Eqs. (1)-(6). The validity of this asymptotic expansion will be assessed a posteriori: once the asymptotic expansion is performed and the row of approximations is taken back.
We consider the time-Fourier transform of Eqs. (1)(6) to perform the asymptotic expansion [see Fedorov (2010)]. In Fourier space (we omit notation changes), the solutions of Eqs. (1)-(6) read as follows:

$$
\begin{aligned}
K & =A(x) \exp (-r x) \\
R & =B(x) \exp (3 r x) \\
r & =(-i \omega+\varepsilon) / c \\
A(x) & =\int_{0}^{x} F_{K} \exp (r s) d s+\gamma B_{0} \\
B(x) & =-\int_{0}^{x} 3 F_{R} \exp (-3 r s) d s+B_{0}, \quad \text { and } \\
B_{0}= & \frac{1}{1-\exp [\ln (\gamma \eta)-4 r L]}\left[\int_{0}^{L} 3 F_{R} \exp (-3 r s) d s\right. \\
+ & \left.\eta \exp (-4 r L) \int_{0}^{L} F_{K} \exp (r s) d s\right]
\end{aligned}
$$

where $i$ is the complex number and $B_{0}=B(0)$. To perform the asymptotic expansion, we use $\exp (a) \approx 1+a$, where $a \ll 1$ is for example $a=r x, a=-3 r x, a=4 r L$, or $a=\ln (\gamma \eta)-4 r L$. We obtain the following system in Fourier space:

$$
\begin{gathered}
K=(3 x / 4 L)\left(f_{K}-f_{R}\right)-(x / 4 L) \ln (\gamma \eta) B_{0}+B_{0} \\
R=(3 x / 4 L)\left(f_{K}-f_{R}\right)+(3 x / 4 L) \ln (\gamma \eta) B_{0}+B_{0},
\end{gathered}
$$

where $B_{0}$ is a zonal-mean component at the first order of the asymptotic expansion. Terms in $x / L$ are a tilting component at the second order. We must, however, retain the second order because it accounts for spatial variability. Consistent with the asymptotic expansion, we choose to remove the zonal-mean component at the second order, because it has the spatial structure of the first order. This implies modifying $x / L$ into $(x / L-0.5)$. We also choose to remove terms depending on $B_{0}$ at the second order. The system now reads as follows:

$$
\begin{gathered}
K=(x / L-0.5)(3 / 4)\left(f_{K}-f_{R}\right)+B_{0} \text { and } \\
(-i \omega+\sigma) B_{0}=(c / 4 L)\left(f_{K}+3 f_{R}\right),
\end{gathered}
$$

with $K=R$. By performing the inverse Fourier transform, one obtains the asymptotic model. The term $-i \omega$ is replaced by $\partial_{t}$. 

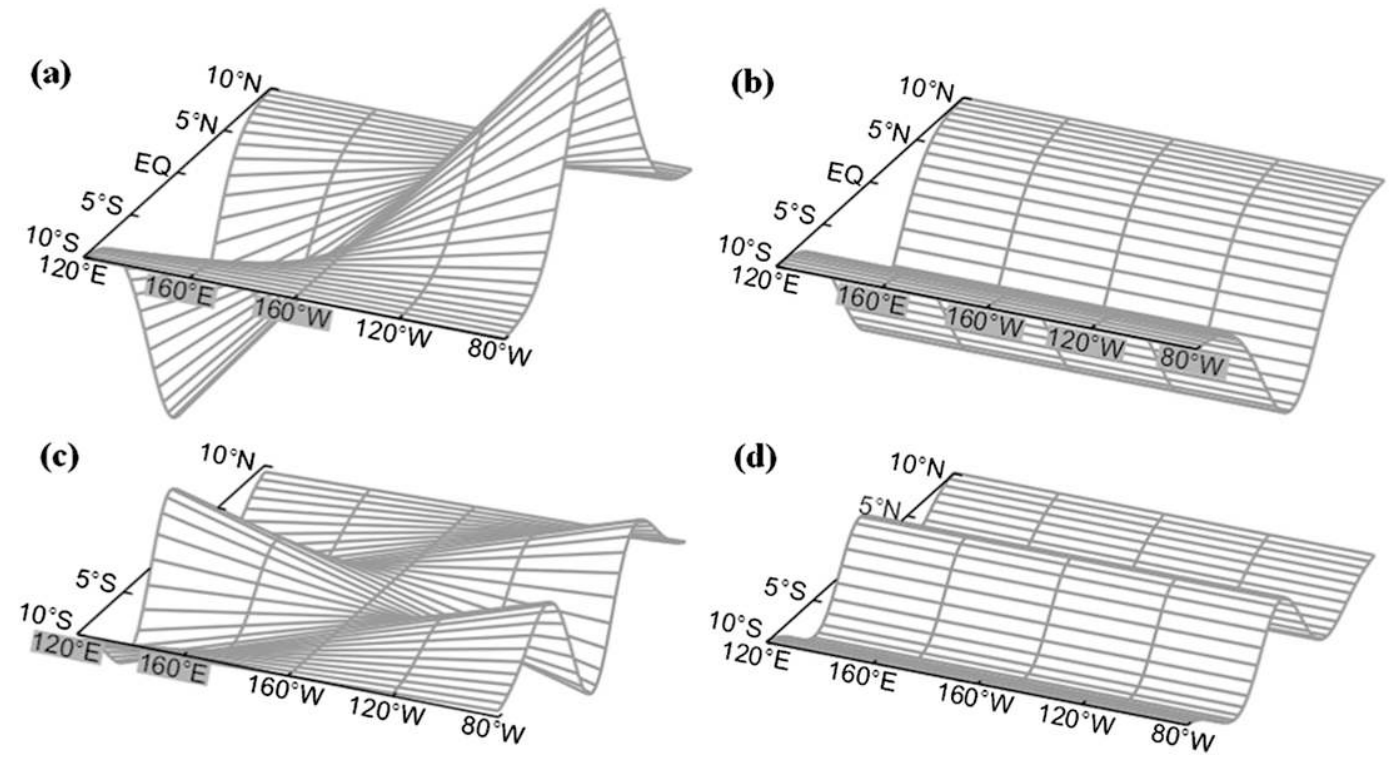

FIG. 3. Nondimensional spatial structure of thermocline depth anomalies from the TW model as a function of lat and lon. (a) Tilt mode structure on $\varphi_{0}$. (b) WWV mode structure on $\varphi_{0}$. (c) Tilt mode structure on $\varphi_{2}$. (d) WWV mode structure on $\varphi_{2}$. We consider the contribution of the gravest ocean baroclinic mode $(n=1)$.

\section{TW model}

From now on, we have finished the asymptotic expansion and take back the initial row of approximations. This simply consists of reconsidering reference values of system parameters $\left(\partial_{t}, \varepsilon, \gamma, \eta\right)$ and forcings $\left(f_{K}, f_{R}\right)$ estimated from observations. We obtain an asymptotic model for the thermocline adjustment to wind stress forcing, the TW model. From Eqs. (7), (22), and (23), thermocline depth anomalies for the baroclinic mode considered (we omit the $n$ notation) read as follows:

$\frac{h}{s c^{2}}=\left[\left(\frac{x}{L}-0.5\right) h_{\mathrm{TILT}}+h_{\mathrm{WWV}}\right]\left(\varphi_{0}+\frac{\varphi_{2}}{2 \sqrt{2}}\right)$,

where $h_{\text {TILT }}$ and $h_{\mathrm{WWV}}$ are scalar functions of time. Thermocline depth anomalies decompose into a Tilt mode and a WWV mode. The amplitude of the Tilt mode is $h_{\text {TILT }}$ and the amplitude of the WWV mode is $h_{\mathrm{wwv}}$. Their temporal evolutions read as follows:

$$
\begin{aligned}
h_{\mathrm{TILT}} & =(3 / 2)\left(f_{K}-f_{R}\right) \quad \text { and } \\
\left(\partial_{t}+\sigma\right) h_{\mathrm{WWV}} & =(c / 2 L)\left(f_{K}+3 f_{R}\right) .
\end{aligned}
$$

The spatial structure of the Tilt and WWV modes is shown in Fig. 3. The Tilt mode consists of a zonal tilt of thermocline depth anomalies, while the WWV mode is constant zonally. This decomposition of thermocline anomalies into a tilting and zonal-mean component is also encountered in previous studies (Jin 1997a,b;
Fedorov 2010; Clarke 2010). Here, the meridional structure is particular and is given by the gravest Hermite functions. The contribution of the second Hermite function $\varphi_{2}$ is somewhat secondary and can be omitted.

The temporal evolution of the Tilt and WWV modes is as in the recharge-discharge model from Jin (1997a,b). One may interpret the Tilt mode as a dynamical mode adjusting very rapidly to wind stress forcing (approximated here as adjusting instantly), and the WWV mode as a dynamical mode adjusting slowly to wind stress forcing with dissipation rate $\sigma$ (Burgers et al. 2005). Consistently with Jin (1997a,b), Fedorov (2010), and Clarke (2010), the WWV mode arises from the propagation and reflection of equatorial waves that are not in phase with wind stress forcing. Here, $\sigma$ is the same as the one solved without wind stress forcing in Eqs. (8)-(11), which is consistent because the WWV mode is obtained from a linear composition of those solutions.

The WWV mode is forced explicitly by wind stress curl (Clarke et al. 2007; Fedorov 2010). Using the property of Hermite function derivatives (e.g., Clarke 2008 , chapter 3) that

$$
l d_{y} \varphi_{1}=-\varphi_{2}+\varphi_{0} / \sqrt{2}
$$

and combining Eqs. (3), (4), and (12), we can rewrite Eq. (26) into

$$
\left(\partial_{t}+\sigma\right) h_{\mathrm{WWV}}=(c / 2 L) f_{\mathrm{CURL}} \quad \text { and }
$$




$$
f_{\mathrm{CURL}}=P \frac{\int_{-\infty}^{+\infty} \int_{0}^{L}\left(\partial_{y} \tau\right) \varphi_{1} d x d y}{\sqrt{2} \rho c^{2}},
$$

where $f_{\text {CURL }}=f_{K}+3 f_{R}$ is the zonally integrated projection of wind stress curl on $\varphi_{1}=\psi_{1}(y / l)$.

For each baroclinic mode, we define an integrated energy as follows:

$$
E=\int_{0}^{L} \int_{-\infty}^{+\infty} \frac{h^{2}}{2} d x d y .
$$

For this integrated energy, the contribution of the Tilt mode may be approximately omitted. In this case, the energy budget reads as follows:

$$
\left(\partial_{t}+2 \sigma\right) E=\Gamma h_{\mathrm{WWV}} f_{\mathrm{CURL}},
$$

where $\Gamma$ is a constant deduced from the problem. This energy budget shares some similarities with the one of Fedorov (2007, its appendix B) considered on the longwave equations [see also Brown and Fedorov (2010b), their appendix B]. Here, the energy losses are explicit and arise from friction and boundary reflections.

\section{Comparison with observations}

We compare the TW model solutions with observations from the Simple Ocean Data Assimilation (SODA) reanalysis (Carton and Giese 2008) over the period 19582007. Figure 4 shows the evolution of thermocline depth anomalies in the equatorial Pacific Ocean, from the SODA reanalysis (Fig. 4a), the long-wave Eqs. (1)-(6) forced by SODA winds (Fig. 4b), and the TW model forced by SODA winds (Fig. 4c). The long-wave Eqs. (1)(6) that are forced by SODA winds accurately reproduce the thermocline depth variability, indicating that the initial truncation of ocean dynamics (first equatorial waves and baroclinic modes) is appropriate. Because of its simpler spatial structure, the TW model only accurately reproduces the large-scale thermocline depth variability. Its solutions slightly lead the observed signal, probably because of the low-frequency approximation that neglects the propagation time of equatorial waves for the adjustment of the Tilt mode.

We further compare the TW model solutions with observations using a Tilt mode index $I_{\text {TILT }}$ and a WWV mode index $I_{\mathrm{WWv}}$. They read $I_{\mathrm{TILT}}=\left(I_{E}-I_{W}\right) / 2$ and $I_{\mathrm{WwV}}=\left(I_{E}+I_{W}\right) / 2$, where $I_{E}$ and $I_{W}$ are averages of $H$ within $160^{\circ}-80^{\circ} \mathrm{W}$ and $120^{\circ} \mathrm{E}-160^{\circ} \mathrm{W}$, respectively, and within $5^{\circ} \mathrm{N}-5^{\circ} \mathrm{S}$. The $I_{\mathrm{TILT}}$ measures the zonal tilting of the thermocline. The $I_{\mathrm{WwV}}$ measures the zonal-mean thermocline depth and is also the conventional WWV index (Meinen and McPhaden 2000). This division of the equatorial Pacific Ocean into a western and eastern half is also considered in the recharge-discharge model (Jin $1997 \mathrm{a}, \mathrm{b})$. Figure 5 shows evolution of $I_{\mathrm{TILT}}$ and $I_{\mathrm{WWV}}$, which are estimated from the SODA reanalysis (black) and from the TW model forced by SODA winds (red). The TW model solutions are in overall agreement with observations (correlations are 0.89 and 0.73 for $I_{\text {TILT }}$ and $I_{\mathrm{WWV}}$, respectively), despite the model simplicity [also see Fig. 9 of Fedorov (2010) for a similar diagnostic]. To compute Fig. 5, we have slightly increased the western boundary reflection coefficient $\gamma$ from 0.5 to 0.7 , which gives the best match to observations (the same values are also used in Fig. 4). This slight heuristic adjustment is not obligatory in the TW model; however, it corrects an artificial source of dissipation. The Kelvin wave resulting from the reflection at the western boundary is slightly too weak, which is likely caused by the omission of higher Rossby waves.

\section{Discussion}

An asymptotic model (the TW model) has been derived from the long-wave equations. It describes the slow thermocline response to wind stress forcing in the equatorial Pacific. Despite its simplicity, the asymptotic model is nonheuristic. It captures the essential dynamics thanks to an asymptotic expansion with an approximation of large spatial and temporal scales, and of small dissipation.

The TW model is, in essence, the ocean component of the recharge-discharge model (RD model) from Jin (1997a,b). However, the RD model's truncation of the initial long-wave equations is different, with vertically a two-layer ocean and meridionally a two-strip approximation. Jin (1997b) proposes two derivation methods for the RD model, a two-box approximation [its Eq. (3.2)] or a low-frequency approximation based on delay equations with no friction [its Eq. (5.8)]. A third derivation method for the RD model could be found using the same asymptotic procedure as in the present study, starting with Eqs. (2.4)-(2.6) from Jin (1997b) that consist of an eastward- and westward-propagating Riemann invariant with associated boundary reflections. For this reason, the RD and TW model can be seen as having similar dynamics. Since its formulation by Jin (1997a,b), the RD model has allowed for a very broad range of applications because of its synthetic representation of the slow ocean adjustment. Because the TW model is in essence the ocean component of the RD model, though with some notable differences, perspectives for future work may be considered within a similar line of study. A first simple step to implement is the coupling to 
(a) SODA

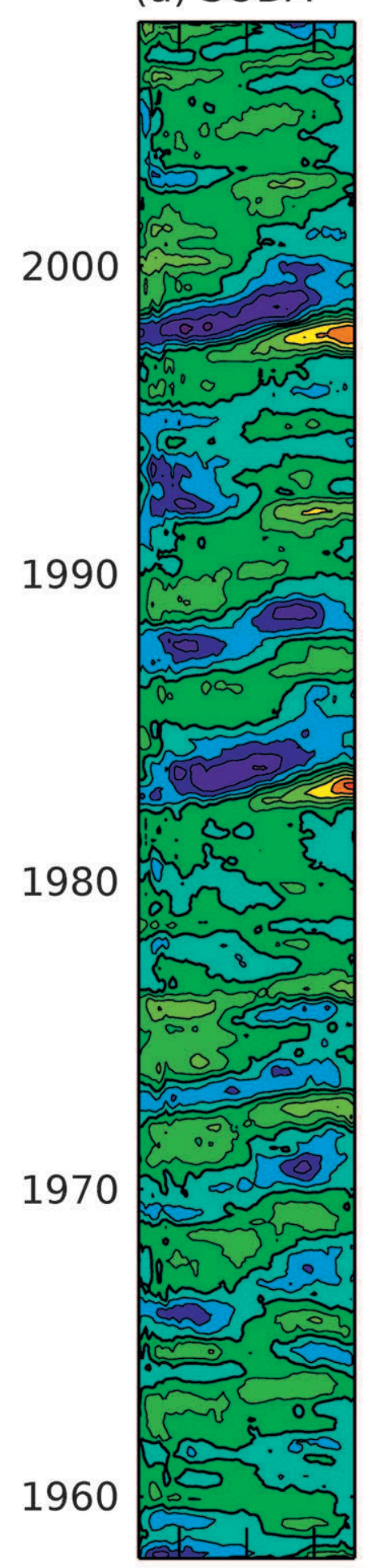

(b) WAVES
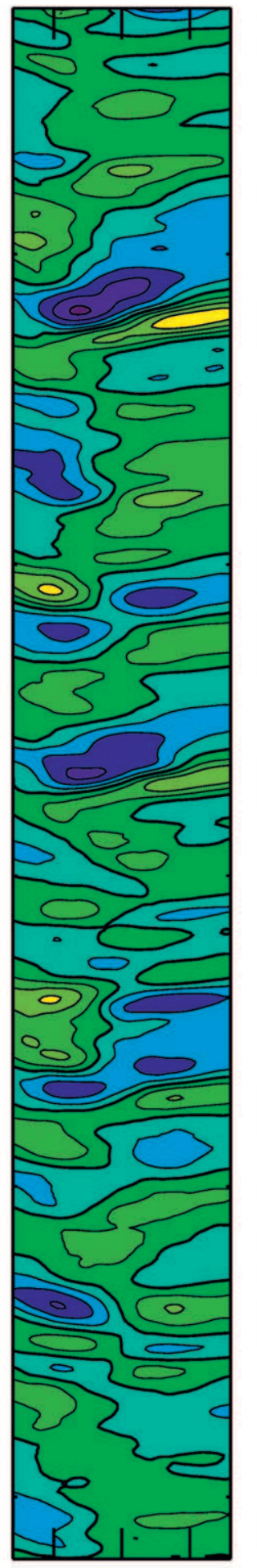

(c) TW model
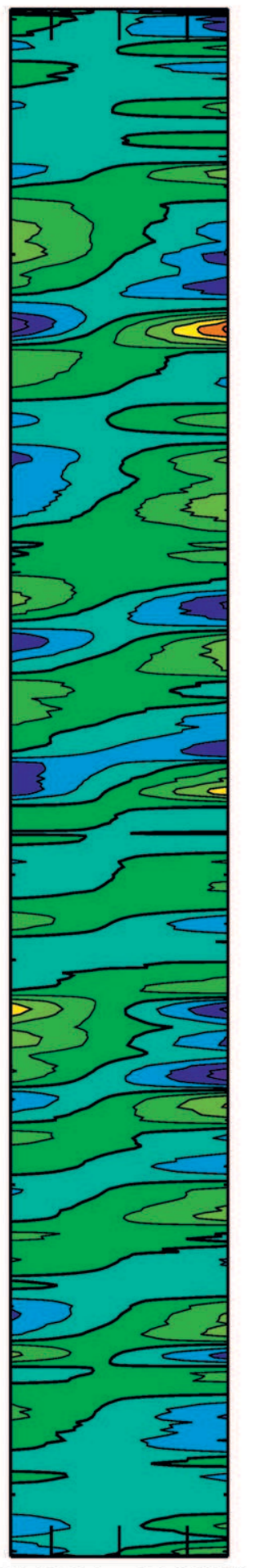

$150^{\circ} \mathrm{E} 110^{\circ} \mathrm{W}$

FIG. 4. Hovmöller diagram of thermocline depth anomalies (average $5^{\circ} \mathrm{N}-5^{\circ} \mathrm{S}$ ) for (a) the SODA reanalysis, (b) the long-wave Eqs. (1)-(6) forced by SODA winds, and (c) the TW model forced by SODA winds (see text). Contour interval is $10 \mathrm{~m}$ and the thick line (from blue to green) is the zero contour. Time series of thermocline and wind stress forcing were detrended and smoothed at 6 months with the time mean removed. 
(a) $\boldsymbol{I}_{T I L T}$

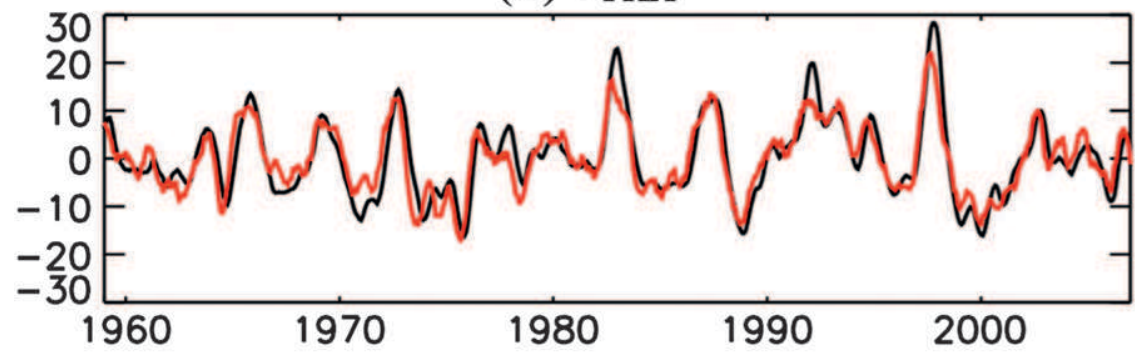

(b) $I_{W W V}$

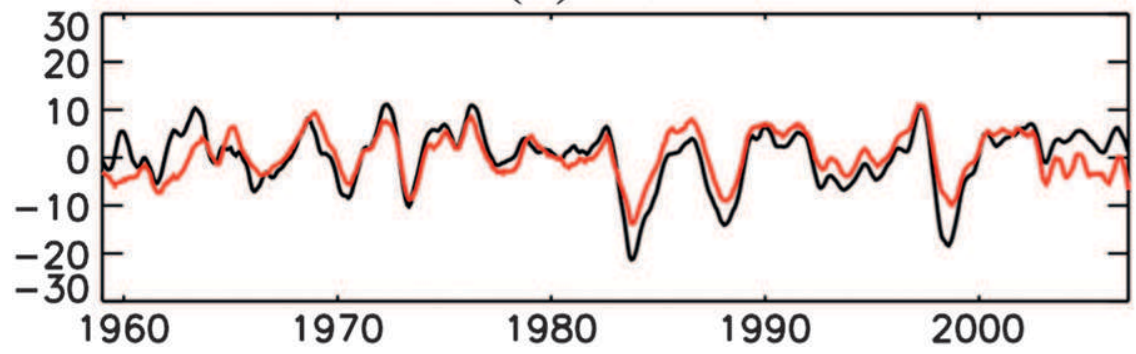

FIG. 5. Time series (m) of (a) $I_{\mathrm{TILT}}$ and (b) $I_{\mathrm{WWV}}$ measured in the SODA reanalysis (black) and in the TW model forced by SODA winds (red). Correlation between time series is 0.89 in (a) and 0.73 in (b). Time series of thermocline and wind stress forcing were detrended and smoothed at 6 months with the time mean removed.

the SST and the atmosphere, in order to invoke ENSOlike oscillations (Jin 1997a,b; An and Kang 2000; An and Jin 2001; Burgers et al. 2005; Clarke et al. 2007; Thual et al. 2011).

In the present study, the long-wave equations are truncated at the equatorial Kelvin and first-order Rossby waves and at the three first baroclinic modes (see also An and Kang 2000; Dewitte 2000; Thual et al. 2011). In particular, the low-frequency approximation considered here is a large-scale approximation: high-order Rossby waves are omitted because they describe smaller meridional scales and zonal scales. At first order, we suppose that they are interfering or dissipating with no upscale contribution to the large-scale flow. Note that a similar argument is commonly considered to omit high-order baroclinic modes (e.g., in two-layer models). Here, to select the equatorial wave and baroclinic mode truncations, we have retained only the fastest propagations of Rossby waves: this phase speed is in $-c_{n} /(2 m+1)$, and this shows a slower decay on the first baroclinic modes with order $n$ than on the first Rossby waves with odd order $m$ (Rossby waves with an even order $m$ are omitted as they do not reflect onto Kelvin waves). The first baroclinic modes are also worth considering because they have a similar large-scale vertical structure with respect to the upper ocean ( $\approx 300$ first meters), and because they project a significant amount of momentum from wind stress forcing (Dewitte 2000; Thual et al. 2011). The present asymptotic expansion could nevertheless be extended to higher-order Rossby waves (at least a few) for more accuracy. Notably, this may correct a slight artificial source of dissipation (on $\gamma$ ) arising from a too weak Kelvin wave reflected at the western boundary (cf. the previous section).

A Tilt and WWV mode with similar features to the present study are also found in the asymptotic model from Fedorov [2010, the first and second terms in the right-hand side of its Eq. (4.1), respectively] and the Fourier solutions from Clarke [2010, its Eq. (5.4)]. Those studies also consider a low-frequency approximation to expand the long-wave equations in Fourier space: the most notable differences with the present study are that the flow is not truncated meridionally (though there is an assumption of the meridional shape of wind stress forcing), and that the energy leakage at the western boundary (i.e., the no-net-flow condition) is retained when performing the asymptotic expansion. As a result, the slow thermocline adjustment in Fedorov (2010) is described by a richer integro-differential equation, which can be approximated to describe delayed propagations as well. In Clarke (2010), the meridional dependency of westward propagation speed (i.e., the higher Rossby modes) is found to be essential to the WWV mode disequilibrium, in discrepancy with the present study.

In the TW model, the WWV mode is explicitly forced by wind stress curl. Clarke et al. (2007) has found a 
similar relation using a box average of thermocline anomalies. Fedorov (2010) has also evidenced this sensitivity using a prescribed wind stress forcing with curl parameter. In the TW model, due to the equatorial wave truncation, wind stress curl projects on the first asymmetric Hermite function (i.e., $\left.\varphi_{1}\right)$. Wind stress curl that forces the WWV mode is nonessential at the equator where $\varphi_{1}$ is zero, and is essential off-equator where $\varphi_{1}$ is maximal (around $\pm 3^{\circ} \mathrm{N}$ for the first baroclinic mode). In comparison, wind stress that forces the Tilt mode is essential at the equator (where $\varphi_{0}$ is maximal). This calls for a further exploration of the role of off-equatorial wind stress curl on the WWV mode (e.g., of the intertropical convergence zone).

Here, the Tilt mode sloping is rather simple (constant with $x$ ), because of the approximation of zonally constant wind stress forcing. In observations (Fig. 1), the sloping is rather confined to the central Pacific where wind stress forcing is maximal. To account for this, one could derive the TW model with nonzero wind stress forcing over a restricted domain [e.g., from $1 / 4$ to $3 / 4$ of the basin length as in An and Jin (2001)]. One could also adequately relax the assumption of zonally constant wind stress forcing once the TW model is derived (e.g., replace $f_{K}(x / L-0.5) \approx \int_{0}^{x} F_{K} d s-0.5 \int_{0}^{L} F_{K} d s$ for the Tilt mode). A similar treatment may be found to account for the WWV mode's zonal dependency in observations (Fig. 1).

The low-frequency approximation considered here has implications for interpreting the slow thermocline adjustment, or "ocean memory," that is essential to ENSO (Neelin et al. 1998). The time delay of equatorial wave propagation has been considered in various studies to be the building element of ocean memory. This interpretation is best illustrated by the delayed oscillator (Suarez and Schopf 1988) and related studies. The interpretation here is different. In the low-frequency approximation, the WWV mode or ocean memory integrates the net adjustment from round-trip propagations of equatorial waves across the basin (for which explicit time-delays are nonessentials). This net adjustment is nonzero because the planetary Coriolis force renders eastward and westward propagations asymmetric (boundary reflections are also asymmetric but this is not accounted for in the present asymptotic expansion). This interpretation, in terms of a net adjustment, is best illustrated by the recharge-discharge model (Jin 1997a,b).

Acknowledgments. The authors thank A. Fedorov and an anonymous reviewer for their detailed reading and comments that helped to improve the manuscript, as well as S.-I. An for fruitful discussions. S. Thual has been supported by Centre National de la Recherche Scientifique
(CNRS) and Conseil Régional Midi-Pyrénées under Contract 022009. The authors also acknowledge support from Centre National d'Etudes Spatiales (CNES) (Modokalt project).

\section{REFERENCES}

An, S.-I., and I.-S. Kang, 2000: A further investigation of the recharge oscillator paradigm for ENSO using a simple coupled model with the zonal mean and eddy separated. J. Climate, 13, 1987-1993.

_ , and F.-F. Jin, 2001: Collective role of thermocline and zonal advective feedbacks in the ENSO mode. J. Climate, 14, 34213432.

Bosc, C., and T. Delcroix, 2008: Observed equatorial Rossby waves and ENSO-related warm water volume changes in the equatorial Pacific Ocean. J. Geophys. Res., 113, C06003, doi:10.1029/2007JC004613.

Brown, J. N., and A. V. Fedorov, 2010a: Estimating the diapycnal transport contribution to warm water volume variations in the tropical Pacific Ocean. J. Climate, 23, 221-237.

$\longrightarrow$, and 2010b: How much energy is transferred from the winds to the thermocline on ENSO time scales? J. Climate, 23, 1563-1580.

Burgers, G., F.-F. Jin, and G. J. van Oldenborgh, 2005: The simplest ENSO recharge oscillator. Geophys. Res. Lett., 32, L13706, doi:10.1029/2005GL022951.

Cane, M. A., and S. E. Zebiak, 1985: A theory for El Niño and the Southern Oscillation. Science, 228, 1085-1087.

Carton, J. A., and B. S. Giese, 2008: A reanalysis of ocean climate using Simple Ocean Data Assimilation (SODA). Mon. Wea. Rev., 136, 2999-3017.

Clarke, A. J., 2008: An Introduction to the Dynamics of El Niño and the Southern Oscillation. Academic Press, 324 pp.

, 2010: Analytical theory for the quasi-steady and lowfrequency equatorial ocean response to wind forcing: The tilt and warm water volume modes. J. Phys. Oceanogr., 40, 121-137.

_ S. Van Gorder, and G. Colantuono, 2007: Wind stress curl and ENSO discharge/recharge in the equatorial Pacific. J. Phys. Oceanogr., 37, 1077-1091.

Dewitte, B., 2000: Sensitivity of an intermediate ocean-atmosphere coupled model of the tropical pacific to its oceanic vertical structure. J. Climate, 13, 2363-2388.

Fedorov, A. V., 2007: Net energy dissipation rates in the tropical ocean and ENSO dynamics. J. Climate, 20, 1108-1117.

$\ldots$, 2010: Ocean response to wind variations, warm water volume, and simple models of ENSO in the low-frequency approximation. J. Climate, 23, 3855-3873.

Goddard, L., and S. G. Philander, 2000: The energetics of El Niño and La Niña. J. Climate, 13, 1496-1516.

Jin, F.-F., 1997a: An equatorial ocean recharge paradigm for ENSO. Part I: Conceptual model. J. Atmos. Sci., 54, 811-829. , 1997b: An equatorial ocean recharge paradigm for ENSO. Part II: A stripped-down coupled model. J. Atmos. Sci., 54, 830-847.

- 2001: Low-frequency modes of tropical ocean dynamics. J. Climate, 14, 3874-3881.

Kessler, W. S., 2002: Is ENSO a cycle or a series of events? Geophys. Res. Lett., 29, 2125, doi:10.1029/2002GL015924.

Lengaigne, M., U. Hausmann, G. Madec, C. Menkes, J. Vialard, and J. M. Molines, 2011: Mechanisms controlling warm water volume 
interannual variations in the equatorial Pacific: Diabatic versus adiabatic processes. Climate Dyn., 38 (5-6), 1031-1046.

McPhaden, M. J., 2012: A 21st century shift in the relationship between ENSO SST and warm water volume anomalies. Geophys. Res. Lett., 39, L09706, doi:10.1029/2012GL051826.

Meinen, C. S., and M. J. McPhaden, 2000: Observations of warm water volume changes in the equatorial Pacific and their relationship to El Niño and La Niña. J. Climate, 13, 3551-3559.
Neelin, J. D., D. S. Battisti, A. C. Hirst, F.-F. Jin, Y. Wakata, T. Yamagata, and S. E. Zebiak, 1998: ENSO theory. J. Geophys. Res., 103 (C7), 14261-14290.

Suarez, M. J., and P. S. Schopf, 1988: A delayed action oscillator for ENSO. J. Atmos. Sci., 45, 3283-3287.

Thual, S., B. Dewitte, S.-I. An, and N. Ayoub, 2011: Sensitivity of ENSO to stratification in a recharge-discharge conceptual model. J. Climate, 24, 4332-4349. 